\title{
Liquid-based cytology: the new screening test for cervical cancer control
}

\author{
Euphemia McGoogan, MB ChB, FRCPath, Associate Medical Director and Consultant Pathologist, NHS Lothian University \\ Hospitals Division, Clinical Services Division, Royal Infirmary of Edinburgh and Senior Lecturer, Department of Pathology, \\ University of Edinburgh, Edinburgh, $U K$
}

Correspondence: Dr Euphemia McGoogan, Clinical Services Division, 2nd Floor, Royal Infirmary of Edinburgh, 51 Little France Crescent, Edinburgh EH16 4SA, UK. Tel: +44 (0) 131242 3368. Fax: +44 (0) 1312423370.

E-mail: Euphemia.McGoogan@luht.scot.nhs.uk

(Accepted 11 February 2004)

Journal of Family Planning and Reproductive Health Care 2004; 30(2): 123-125

\section{Background to the UK Cervical Screening Programmes}

On 22 October 2003, the National Institute for Clinical Excellence (NICE) recommended to the National Health Service (NHS) in England and Wales that liquid-based cytology (LBC) should be introduced as the primary means of processing samples in the NHS Cervical Screening Programme (NHSCSP) This followed a decision of the Scottish Minister for Health in April 2002 that LBC should be implemented as the routine screening test throughout Scotland.

Regular screening of a high proportion of the population at risk has been shown to result in a major decrease in the incidence and mortality from cervical cancer in that population. Organised programmes, such as the NHSCSP in the UK, have successfully halved the incidence of cervical cancer by implementing regular screening at 3-5-year intervals for more than $80 \%$ of the population at risk, backed up by quality assurance programmes and health professionals working together as a co-ordinated team. Quinn et al. showed that the invasive cancer rate in the UK dropped by $50 \%$ following the introduction of the organised screening programme in $1988 .^{1}$

\section{The cervical smear test}

The conventional cervical smear test has been used for over half a century. Cells are scraped from the surface of the cervix using a wooden spatula and spread onto a glass slide. The cells are then 'fixed' with a fluid or spray to prevent them degenerating and the 'smear' is sent to the laboratory for assessment. In the laboratory the slide is stained with a series of dyes to make the cells visible under the microscope. Trained staff scan these smears looking for any abnormal cells among the hundreds of thousands of normal cells on each slide. If the preservation is poor, the cells do not stain crisply and are difficult to assess. If there is pus or blood, or the sample is spread too thickly, abnormal cells may be obscured.

Until recently, there was little understanding of the inherent limitations of the cervical smear but we now recognise that the conventional smear test lacks sensitivity, specificity, reliability and repeatability. Analyses of studies in the peer-reviewed literature ${ }^{2-4}$ have repeatedly shown that a smear test on one occasion can only identify just over half the women who have cervical intraepithelial neoplasia (CIN) at that time. The success of the NHSCSP is due to retesting women at frequent intervals to compensate for this low sensitivity. Fortunately it takes on average over 10 years for CIN3 to develop into an invasive cancer during which time a woman may be routinely screened several times.
In the last 15 years there has been increasing concern about false-negative smear test results. An accurate cervical screening test requires that abnormal cells are collected from the cervix and transferred to the glass slide for microscopic review. A failure in either of the first two steps is regarded as a sampling error, while a failure in the laboratory examination is termed a screening error.

Factors such as incorrect or inadequate smear-taking technique and the histological type, site and size of the lesion in the woman's cervix influence the number of cells removed from a suspect area. Furthermore, no matter how expert the smear-taker, not all the cells removed from the cervix are placed on the glass slide. Only a selected (and not randomised) proportion of cellular material is transferred and this may include all, none or just some of any abnormal cells. It has been shown that as many as $90 \%$ of the cells removed from the cervix may be discarded with the sampler. 5 Slides too thickly spread or inadequately fixed cannot be optimally stained in the laboratory. This in turn limits the quality of the microscopic slide assessment. Publications suggest that the relative proportion of sampling to screening errors is about $2: 1.5$

However, false-negative results are not the only problem facing organised screening programmes. In order to decrease the false-negative rate, many laboratories have increased their false-positive rate (i.e. the proportion of smears called inadequate and borderline). As a result, fewer and fewer women with normal cervices are being given a normal smear test result.

In the UK, where the routine screening interval is up to 5 years, the criteria used for assessing adequacy are more stringent than those used in countries where women are retested annually. It is therefore not surprising that the inadequate rate in the UK is higher than in the USA. However, the inadequate rate has been increasing steadily over the last 10 years to a current average of over $9 \%$. Thus, up to 1 in 10 women attending for screening must return for a repeat smear simply because the first test was unsatisfactory for interpretation.

There are economic and emotional costs associated with increased referrals for repeat smears or further investigation. The women involved may suffer prolonged anxiety; and primary care teams are unnecessarily burdened with extra work, as are computerised recall systems and laboratories. All this in turn increases the cost of the NHSCSP. Improvements in the sensitivity and specificity of the routine screening test had to begin with improved techniques of specimen collection to allow better quality samples, better slide preparations for microscopic assessment and the potential for ancillary tests.

Having achieved a major improvement in the coverage and quality of the NHSCSP by 1998 , attention turned to 
improving the quality of the screening test itself. It was suggested that the deficiencies of the conventional smear could be addressed by using liquid-based preparation technology.

\section{What is LBC technology?}

In LBC technology, a Cervex-Brush ${ }^{\circledR}$ (Rover's Medical Devices BV, Oss, The Netherlands) or a combination of a plastic spatula and endocervical brush are used to collect the sample. Instead of spreading the cellular material removed from the cervix onto a glass slide, the samplers are rinsed, or the head of the broom is detached, into a vial of liquid transport medium creating a cell suspension. This cell suspension contains all of the cells removed from the cervix and will remain well preserved for several weeks at room temperature.

In the laboratory excess blood or inflammatory exudate are removed and a small representative aliquot of epithelial cells is deposited in a thin layer within a circle on a glass slide. The cells on the slide are therefore a proportional representation of the range of epithelial cells in the original sample. These preparations result in fewer unsatisfactory test results since the cells are well preserved and clearly visualised. Abnormal cells are not hidden in thick areas of the slide even when they are very few in number or very small in size. These LBC slides are quicker and easier to screen than conventional smears and can increase the laboratory throughput by up to $40 \%$.

In addition, there are sufficient cells left in the suspension for additional tests, e.g. human papillomavirus (HPV) DNA, other microbiological infections such as chlamydia, in addition to a whole range of molecular tests that may, in the future, prove to be of value in predicting which women are more at risk of developing cervical cancer.

There are two devices commercially available to prepare LBC cell suspensions in the laboratory: ThinPrep ${ }^{\circledR}$ (Cytyc UK Ltd, Crawley, UK) and SurePath ${ }^{\text {TM }}$ (TriPath Imaging, Inc., Burlington, NC, USA) formerly AutocytePrep ${ }^{T M}$ or CytoRich ${ }^{\circledR}$. These systems are also the most widely studied technologies in the literature. However, they involve different technologies and require differing laboratory resources. The main difference for the smear taker is that ThinPrep requires the sampling device(s) to be rinsed in the preservative collection fluid while, by contrast, the head of the Cervex-Brush is broken off into the vial for the SurePath system. Clinicians must clearly understand which system their laboratory is using since the wrong preparation method invalidates the sample. Both systems have Food and Drug Administration (FDA) approval for routine cervical screening and currently LBC constitutes over $75 \%$ of cervical screening tests performed in the USA. LBC is also widely used in other parts of the world including Western Europe, Australia and New Zealand.

\section{The NHSCSP HPV/LBC pilots}

The NHS is committed to evidence-based medicine and new drugs and technologies must be shown to be cost effective. Payne et al., ${ }^{2}$ in a report commissioned by NICE, showed that prior to the year 2000, there were no published studies to provide direct evidence regarding the costeffectiveness of LBC for cervical screening in the UK. However, the report suggested that LBC would reduce both the number of inadequate samples and false-negative results and decrease the time required for examination of specimens by cytologists. NICE immediately commissioned three English pilots of LBC to confirm the cost effectiveness of the technique. NICE also recommended that the NHS should take the opportunity provided by these pilots to evaluate the role of reflex HPV DNA testing of cervical samples with low-grade abnormalities.

Three English laboratories were chosen for these pilots: Bristol and Norwich used ThinPrep while Newcastle converted to the SurePath system. A total of 100000 routine screening tests were collected and the LBC results were published in 2003. 6 The HPV arm of the pilots (reflex HPV testing on the residual cells in the vial for borderline and mild dyskaryosis cytological results) required a further year to collect follow-up information and is expected to report in late spring 2004.

The LBC arm evaluation showed an $80 \%$ reduction in the inadequate rate and a reduction from $5.4 \%$ to $4.6 \%$ in the overall rate of borderline smears. While there was no significant increase in the rates of moderate and severe dyskaryosis when averaged across the three sites, those sites using ThinPrep showed an overall increase in severe dyskaryosis while that using SurePath saw a drop in the detection of severe dyskaryosis. This finding is currently being investigated. The positive predictive value increased, indicating improved accuracy in the diagnosis of high-grade cytological abnormalities at all sites.

A feature that caused concern was a significant decrease in the number of cytological glandular abnormalities reported across all sites in the pilot. However, the number of women detected at colposcopy with cervical glandular neoplasia increased during the same period. Thus it is suggested that LBC allows for more accurate morphologic typing of abnormalities than the conventional cervical smear.

The report suggested that workload for laboratories and primary care was likely to decrease. The difference in inadequate rates, with a possible further slight reduction due to fewer borderline smears, should result in fewer tests being done. Referrals to colposcopy are likely to be affected only if the overall reporting of highgrade lesions increases. It was concluded that there was robust evidence that $\mathrm{LBC}$ was a cost-effective alternative to conventional smears, especially in terms of life years saved.

\section{Cervical Screening Programmes in Scotland and Wales}

The NHS in Scotland took a different approach. In 2001, a LBC pilot was established in four laboratories (Airdrie, Dundee, Aberdeen and Inverness) with very different service profiles. It was decided that all laboratories should use the ThinPrep system since Cytyc had a more established infrastructure to support the laboratories at that time. A total of 30000 routine screening tests were collected and the results were published by the Scottish Executive Health Department in $2002 .^{7}$ The Scottish pilot report also showed a sharp reduction in the unsatisfactory smear rate but a significant improvement in the detection of high-grade lesions (between 3 and 9 women per 1000 tested). Reduced workload and increased productivity were also demonstrated in laboratories.

The Scottish Cervical Screening Programme began converting to LBC in 2002. All Scottish laboratories opted to use the ThinPrep system and Scotland has now fully converted to ThinPrep for its routine screening test.

A pilot of LBC was also implemented in Wales at a similar time to the English pilots; again this pilot only used ThinPrep technology and this is now being rolled out. 
The cost effectiveness of $\mathrm{LBC}$

Until very recently it had been difficult to draw clear conclusions from the available literature as to sensitivity, specificity and cost benefits of LBC due to deficiencies in the study design of the published articles. Most studies used a selected population with a high incidence of disease. Many studies lacked verification of the diagnosis, total study numbers were small and normal results were not verified.

The UK pilots have provided invaluable independent information about the efficacy and cost effectiveness of LBC. The role of $\mathrm{LBC}$ in improving the quality of organised screening programmes is now irrefutable. In October 2003 NICE, having evaluated all the available evidence, recommended converting to $\mathrm{LBC}$ for routine screening in England and Wales. In recognition of the steep learning curve for assessment of LBC slides even for very experienced laboratory staff, NICE waived its normal 3 months' implementation for a 5-year conversion timescale.

\section{The future}

Since these LBC systems can handle very large numbers of samples each year there is a unique opportunity for modernisation of laboratory services through rationalisation of the preparation and staining of LBC samples to a smaller number of centres. Furthermore, since LBC systems deposit cells onto a thin-layer on a microscope slide, computer-assisted imaging is facilitated. Devices have been developed that either identify slides not requiring human review or identify cells on the slide for human assessment (computer-assisted screening). These technologies have the potential to revolutionise cervical screening, because they can decrease the fatigue of the user, allow 50\% more slides to be reviewed per day, decrease the screening error rate and, with appropriate decision support, identify morphologic features that may not be not apparent in routine human review.

Pathologists and clinicians must understand, however, that different liquid-based techniques will yield different results depending on the type of collection device, the type of preservative fluid, the method of dispersing cells in liquid, and the manner in which cells are collected and deposited onto a slide. All liquid-based methods are not necessarily equal in their performance, simply because they are 'liquid based'. There are a variety of laboratory devices other than ThinPrep and SurePath currently under development that will also prepare LBC samples. However, evidence of their ability to deliver an appropriately preserved, representative cell sample onto the glass slide in a consistent manner is not yet available in the peerreviewed literature.

\section{HPV testing}

Certain types of HPV are the primary cause of almost all cervical cancers. HPV DNA is probably the best marker of CIN available at present. HPV testing can be carried out on LBC samples and is more sensitive but less specific than cytology for detecting high-grade CIN, especially for women aged under 30 years. However, the negative predictive value of HPV DNA is extremely high. The NHS pilots are evaluating the use of reflex HPV DNA testing on LBC as a means of managing women with low-grade cytological abnormalities and the results are expected imminently. Using HPV testing as a primary screening approach could probably only be applied to women aged over 30 years and would require efficient management of HPV-positive women with negative or borderline cytology results being returned to routine screening. ${ }^{8}$

\section{Direction of further research}

Further research is needed in understanding the interrelationship between morphologic cellular appearances and the presence of HPV within cells, or the molecular alterations in the cell that arise from HPV infection pushing the cell towards neoplasia. The residual material from the LBC cell suspension may be an invaluable research resource for the further investigation of molecular markers that may prove useful in the future.

Statements on funding and competing interests

Funding. The author has received grants and conference expenses from a variety of companies involved in cervical screening technology including Digene, Cytych and Autocyte.

Competing interests. None identified.

References

1 Quinn M, Babb P, Jones J, et al. Effect of screening on incidence and mortality from cancer of the cervix in England: evaluation based on routinely collected statistics. BMJ 1999; 318: 904-908.

2 Payne N, Chilcott J, McGoogan E. Liquid-based Cytology in Cervical Screening: A Rapid and Systematic Review. Southampton, UK: The National Co-ordinating Centre for Health Technology Assessment, 2000.

3 Fahey MT, Irwig L, Macaskill P. Meta-analysis of Pap test accuracy. Am J Epidemiol 1995; 141: 680-689.

4 American College of Obstetricians and Gynecologists (ACOG) Committee Opinion. New Pap test screening techniques. Int $J$ Gynecol Obstet 1998; 63: 312-314.

5 Hutchinson ML, Isenstein LM, Goodman A, et al. Homogenous sampling accounts for the increased diagnostic accuracy using the ThinPrep Processor. Am J Clin Pathol 1994; 101: 215-219.

6 Moss SM, Gray A, Legood R, et al. First report to the Department of Health on evaluation of LBC. 2002. http://www.cancerscreening. nhs.uk/cervical/lbc-pilot-evaluation.pdf

7 Cervical Screening Programme - Liquid-based Cytology (LBC). HDL(2002)61. http://www.show.scot.nhs.uk/publicationsindex.htm.

8 Cuzick J, Szarewski A, Cubie H, et al. Management of women who test positive for high-risk types of human papillomavirus: the HART study. Lancet 2003; 362: 1871-1876.

Associate membership of the Faculty of Family Planning and Reproductive Health Care is open to all nurses with a special interest in contraception and reproductive health. The annual subscription is currently £40. This subscription entitles Associate Members to copies of the Journal of Family Planning and Reproductive Health Care and access to the members' enquiry service. For further information please refer to the Faculty website at www.ffprhc.org.uk (latest updates section). 